\title{
Heart rate variability complexity in the aging process
}

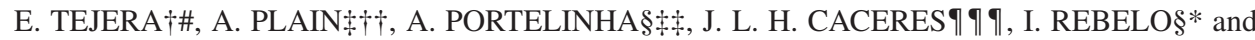 \\ J. M. NIETO-VILLAR $\dagger \| \S \S$ \\ †Dto. de Química-Física, Fac. de Química, Universidad de La, Habana, Cuba \\ \#Fac. de Biología, Universidad de La, Habana, Cuba \\ I Center of Cybernetic Apply to Medicine, Cuba \\ $\S$ Faculdade de Farmácia/IBMC, Universidade do, Porto, Portugal \\ ||Cátedra de Sistemas Complejos "H. Poincaré", Universidad de La, Habana, Cuba \\ (Received 12 January 2007; revised 10 February 2007; in final form 25 October 2007)
}

\begin{abstract}
In the present work, we propose a discrete model to the characterization of heart rate variability (HRV) complexity in the aging process of healthy subjects. We apply the Shannon entropy on the basis of an alternative way to probability calculation and to define a new index taking into consideration the transition probability that is related to the physiological complexity of the system. Our results suggest that in the aging process the capability response decreases according to the reduction of the physiological complexity. In the oldest group, an alternative mechanism emerges to compensate for this lack of capability; however, this effect does not increase the physiological complexity. Concomitantly, we provide some physiological explanation for our results.
\end{abstract}

Keywords: Probability; Physiological complexity; Aging process; Capability response; HRV

\section{Introduction}

A biological system can be considered a complex dynamic system changing far from the thermodynamic equilibrium; the complexity and diversity of these systems are the main features that have led researchers to find a multifactorial theory for aging. The complex nature of aging is the main difficulty preventing a single approach. There are several theories like Harman's free radical theory [1,2] that consider several facts, but the factors that determine aging represent one of the fundamental problems of human knowledge.

The cardiovascular system response is related to a great variety of processes (respiratory cycle, hormonal fluctuation, physical and psychological stress, etc.) converting the cardiovascular system in a potential candidate for the study of complex behavior in the organism like the aging process. On the other hand, many of the necessary data to study this system could be obtained with non-invasive techniques that allow checking the system

\footnotetext{
*Corresponding author. Email: irebelo@ff.up.pt \#Email: edutp00@yahoo.com

† Email: merlin@fbio.uh.cu

†tEmail: aportelinha@ @ff.up.pt

ๆ ๆ Email: cacerjlh@ cecam.sl.cu

§ミmail: nieto@fq.uh.cu
} 
response from minutes to days. The HRV study is one of the possibilities and the problem is subsequently the characterization of this signal.

Many indexes had been proposed in these years following the linear and non-linear tools [3-6], but we still need appropriate indices that explain the changes in HRV with age and sex, and that allow a correct prediction according to a physiological approach.

Several problems are present in the study of HRV: the lack of physiological sense of the indexes, the problem with the recording time or series size, the non-stationary behavior of signal, noise, etc. However, some magnitudes like fractal dimension, and entropies by different methods, have shown very good results because they are related to the physiological complexity and in some cases show more information than lineal index (Pnn50, SD1, etc.) [3,5-11].

It is important to note that some indexes are related to the mathematical complexity (ex: predictability) but not with the physiological complexity. For this reason they fail in the study of some diseases and even in the aging process [33].

In this work, we propose a discrete model to the characterization of HRV complexity in the aging process of healthy subjects. We used the Shannon entropy on the basis of an alternative way to probability calculation and define other indexes that are related to physiological complexity and transition space. Concomitantly, we provide some physiological explanation for our results.

\section{Methods}

\subsection{Data collection}

We studied a group of 73 healthy volunteers distributed in four different age groups: $26-30$, 31-40, 41-50 and 51-60 years old, without any kind of pharmacological treatment. The ECGs (electrocardiographic records) were recorded in relaxed sitting positions for a $20 \mathrm{~min}$ period, and the signal digitalized to $40 \mathrm{kHz}$ and $8 \mathrm{bit}$. The filter used in the analysis was proposed by Machado et al. [13]. We eliminate the first $2.5 \mathrm{~min}$ (see result and discussion) of the record and the final RR series had a time interval of $10 \mathrm{~min}$.

\subsection{Theoretical support}

From physiological perspective, RR interval variation is a consequence of the change in sympathetic and/or parasympathetic systems and other events like respiratory rhythms, electrical conduction mechanism, psychological and endocrinal influence, etc. [3,15,19-21,33]. Several described methods consider that RR intervals or RR series are a continuum of values in time, but according to the fact that the $R$ wave is the result of electrical impulses it would be more interesting to interpret variation behavior as a discrete process in the continuo time, as referred to in [18].

The application of discrete models to the study of time series in the scientific literature has been carried out with diverse tools like symbolic dynamic, point process, statistical physics, stochastic approach, etc. [6,14-20]. Some of them take in to consideration different ways to express the probability. The probability function and methods used to evaluate it will reflect some specific interest according to a mathematical or physiological perspective. To construct our discrete model, we considered the following postulates:

1. The RR interval corresponds to a measure of a discrete variable and its possible values do not have the same probabilities.

2. Each measure $\left(E_{i}\right)$ describes a state of the system. 
3. The states numbers $(n)$ remain approximately constant after a certain time and during a period of time if the system is not perturbed.

Due to the complexity of the control mechanism in the RR variation as a consequence of their multiple factors, a state will be the global response of the system to all these variables. If each measure represents a state of the system, then is important to define how many different states are in the RR series. To do this, we use a measurement interval $\Delta E$ (a bin) and the state is characterized by the mean value of the bin $\bar{E}_{i} \pm \Delta E$. This is a clustering process in a time series and other authors have reported good results with a bin of $8-15 \mathrm{~ms}[3,18-21,34]$.

According to the third postulate, the states number $(n)$ must remain approximately constant after a certain time, therefore the bin selected should allow this, and is important to consider that of small window size would result in a fluctuation at the states number associated with the noise of the series, and with the use of higher values we can lose information for the states superposition.

The probability of a specific state in a time interval may be defined as:

$$
P_{i}=\frac{N_{i}}{\sum_{i=1}^{n} N_{i}},
$$

where ' $n$ ' is the states number and $N_{i}$ is the frequency of the state ' $i$ ' in the time interval analyzed. With this probability definition, we are not assuming any specific distribution; it is a simple way to calculate probabilities. However other authors have used Gaussian distribution function to describe the RR intervals [4,19,22]. Given the probability, we calculate the entropy of Shannon as:

$$
S=-k \cdot \sum_{i=1}^{n} P_{i} \ln P_{i}
$$

where $k=1$ in our calculations.

\subsection{Transition between states}

It is known that RR series are irregular, perhaps due to noise or random components, or due to deterministic chaos, but at same time are present some important short and long-range correlations under different scales or partners that are the source of the fractality or multifractality property of the signal $[7,9]$. This short or long-range (fractal) correlation is somehow broken in aging or disease processes and, in this rupture, the physiological complexity decreases $[32,33]$. The short and long-correlation indicates the 'memory' in the heart response to some external or internal stimulation and could be translated saying that the transition probability from the state $i$ to $j$ is not the same for any state $i$ or $j$ and in general depends on the time involved between transitions. In some way this probability is connected to the physiological complexity. It is the main reason to study transitions.

If $T_{i j}$ is the number of transitions from the states $i$ to $j$ (figure 1), then we define the transition frequency matrix $F(n \times n)$ with element $T_{i j}$. The $F$ matrix could be transformed in a binary matrix $F[1]$ with element 1 or 0 if $T_{i j} \neq 0$ and $T_{i j}=0$, respectively.

It is not difficult to note that the graphic representation of $F$ [1] is similar to a Poincaré map and its spatial representation has a big impact in the physiological implication 


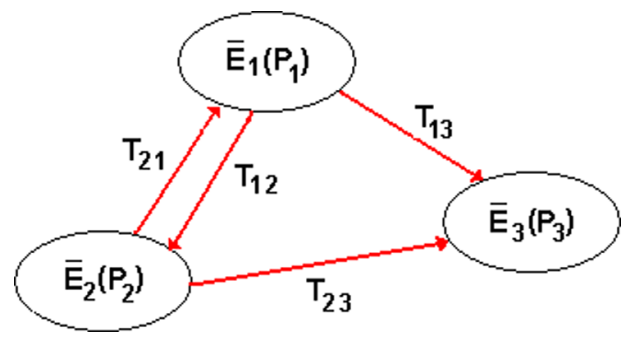

Figure 1. Representation of the model. $\bar{E}_{i}$ is the mean value of RR interval in the state $i$ and $T_{i j}$, is the number of transition from states $i$ to $j$.

[4,23,29-31]. The graphic representation of $F$ (transition maps) has to include a new axis corresponding to the frequency of transition. We will use a color scale to represent this axis.

An important feature of the transition map is its symmetry. If the map is symmetric then $T_{i j}=T_{j i}$ for all $i$ and $j$; on the other hand, if there are preferential states in the system, it is a logical guess that the density of transitions around this states increases and the symmetry has to change. We proposed an asymmetric index $A(F)$ as follows.

If:

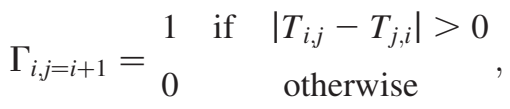

Then, we define:

$$
A(F)=\frac{2}{n(n-1)} \sum_{i}^{n-1} \sum_{j=i+1}^{n} \Gamma_{i j}
$$

The $2 / n(n-1)$ is a normalization term, therefore $0 \leq A \leq 1$. In the cases $A=0$ or $A=1$, the matrix is symmetric or asymmetric, respectively. It is important to emphasize that an increment of the asymmetry values is not necessarily associated with point dispersion in the transition maps but would be a consequence of variations in the density close to a specific area in the map or in the random component of the system dynamic. The asymmetry is related to the roughness of the transition space.

\section{Results and discussions}

In figure $2(\mathrm{~A}),(\mathrm{B})$ it is possible to identify the influence of bin size in the conservation of states numbers and the state of maximal frequency along the recording time. We can observe that with a size of $15 \mathrm{~ms}$, it is possible to obtain a reproducible value in the $n$ and $E_{\max }$ after approximately $2.5 \mathrm{~min}$ of recording time.

Those results do not mean that our series are stationary but they are consistent with the third postulate and at the same time fix the time interval to do our calculations. Above the $2.5 \mathrm{~min}$ approximately (for bin size bigger than $15 \mathrm{~ms}$ ), it is possible to obtain small fluctuations of $n$ and $E_{\max }$; this leads to the possibility of applying our model to extract useful information in short-term recordings that is a general problem in RR time series analysis $[5,9,10,15]$. We select a bin size of $15 \mathrm{~ms}$, but in the range of $10-20 \mathrm{~ms}$ the significance levels between the groups and errors are the same with our indexes. 

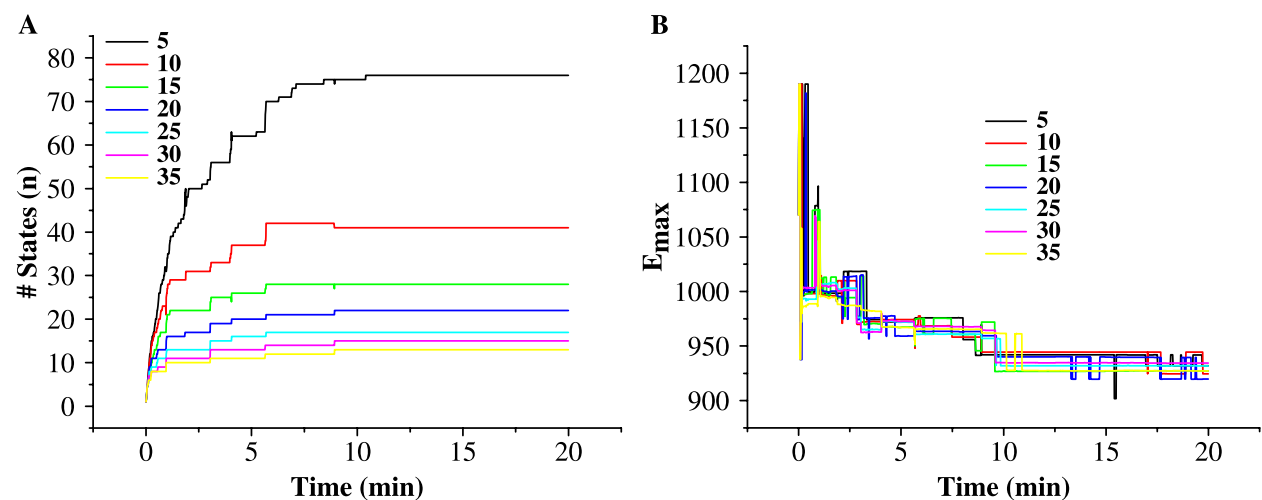

Figure 2. (A) States number $(n)$ and (B) maximal frequency state $\left(E_{\max }\right)$ variation respect to time considering different bin sizes.

The RR mean fluctuation is not significant. This result is not contradictory for two possible reasons: first the hear rate is not only determined by sympathetic modulation-in general it is a result of several complex interactions between sympathetic, vagal, baroreflex and physical influences [23,35]; and second we are using a relatively small population.

The states numbers decrease with the ageing process, and it is evident that there must exist a close relation between this index, SDRR and $S$ (figure 3). The decrement in the heart rate standard deviation (SDRR) is equivalent to the reduction in the states accessibility $(n)$; however this index cannot say anything about how to change the transition between this state, its mean, the $n$ or SDRR index which are independent of the scales correlations.

A little more information is available with the entropy $(S)$. In uncorrelated and uniform state distribution:

$$
S=-\sum_{i=1}^{n} \frac{1}{n} \ln \left(\frac{1}{n}\right)=\ln n,
$$

however in figure 3 the linear equation shows a significant difference with respect to the uncorrelated uniform behavior. The entropies $(S)$ lessen in the aging process (table 1) as we should expect according to previous works $[8,10-12,15,23-25]$ and this decrease
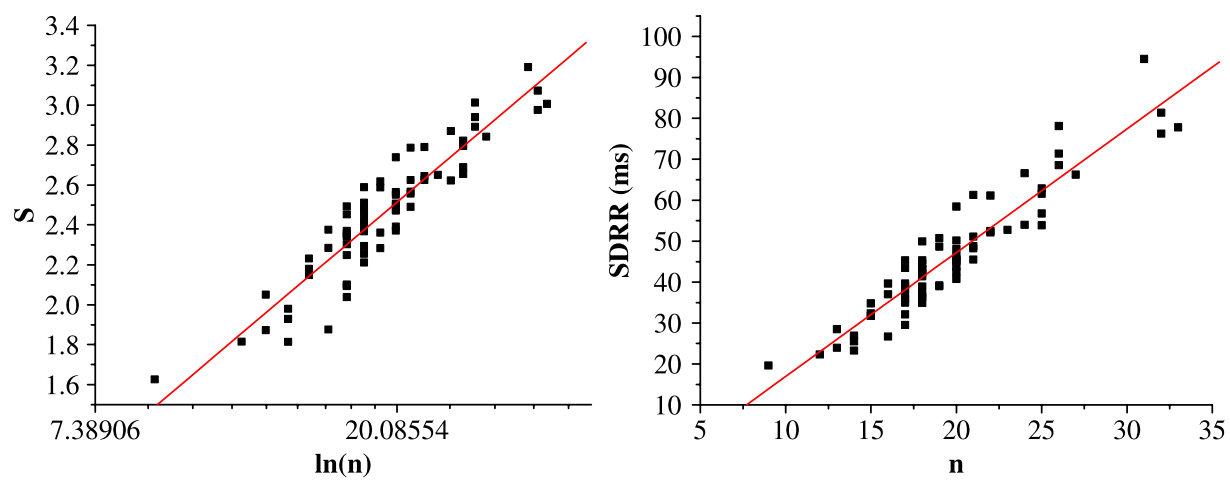

Figure 3. Relationships between entropy $(S)$ and SDRR respect to states number $(n)$. (Left) $S=1.27 \ln (n)-1.3$, $R=0.92, p<0.001$. (Rigth). $R=0.94, p<0.001$. 
Table 1. Mean values of states number $(n)$, entropy $(S)$, asymmetry $(A)$, RR mean, standard deviation of the RR (SDRR) and the low frequency-high frequency ratio form the power spectra ( $\mathrm{LF} / \mathrm{HF})$ obtained from healthy subjects.

\begin{tabular}{lccccc}
\hline Parameters & $26-30$ years & $31-40$ years & $41-50$ years & $51-60$ years & $p$-values \\
\hline$N$ & 24 & 19 & 15 & 18 & $<0.001$ \\
$S$ & 2.73 & 2.52 & 2.15 & 2.34 & $<0.001$ \\
$A$ & 0.33 & 0.34 & 0.31 & 0.28 & $<0.02$ \\
RRmean & 855.41 & 802.00 & 311.04 & 862.52 & $* *$ \\
SDR & 58.98 & 47.60 & 1.542 & 38.70 & $<0.01$ \\
LF/HF & 0.771 & 1.223 & & 1.909 & $<0.01$ \\
\hline
\end{tabular}

**Not significant differences between groups.

is a consequence of the complexity reduction of the signal. In the aging process, several changes in the sympathetic tone had been noted by other authors as an increment in the plasma or epinephrine levels, reduction in the baroreflex sensibility and a reduction in the hear rate variability and SDRR [36,37]. The decrement in the entropy and accessibility space (n) could be related with the same effect and is a signal of the increment in the sympathetic tone. The same reason and the decrement in the physical exercise with the age are the possible causes of the increment in the sympathovagal balance (LF/HF) [3,23,26,28,39].

However, SDRR, $S$ and $n$ indexes show a little increase in the oldest group. This effect is not casual; it has also been described by other authors with a larger population $[23,38]$ and will be discussed in the next topic.

Although the asymmetry would have some mathematical connections with the entropy they represent different information. The entropy is related to the states accessibility; however, asymmetry takes in consideration the transition between these states and decreases in the aging process and most in the oldest group (table 1).

\section{Stability of states}

In a system under certain external conditions that can change and/or modify their dynamic, the states of higher probability correspond to the region of maximal stability, and as a consequence of external influence, the system is forced to transit between different states. For this reason, the capability response or transition space is as important as the region of maximal stability.

The mean probability distribution function of each group (figure 4), shows different behavior according to the results of table 1 . The two youngest groups present similar distribution, but the second one shows a little displacement to lower states indicating an increase in the beat frequency. We can observe in the $41-50$ age group a compression in the states space that must lead to a reduction in the accessible states reflected in the state number and entropy decrease and as a consequence new states appear (close to $1150 \mathrm{~ms}$ ) with relatively low probability. On the other hand, the oldest group shows an increment of states with low beat frequency but with a very low probability.

We can observe in the normalized graphic representation of matrix $\langle F\rangle$ (figure 5) the transition spaces compression process and the appearance of new transition according to the new states in the oldest groups. In this picture, the states are represented by number for simplicity but taking care of scales conservation. As we can verify, a gradual compression of the transition space exists, a clear indication of the reduction in the capability response and an increase of the localization of the transitions (violet area) that could explain the 


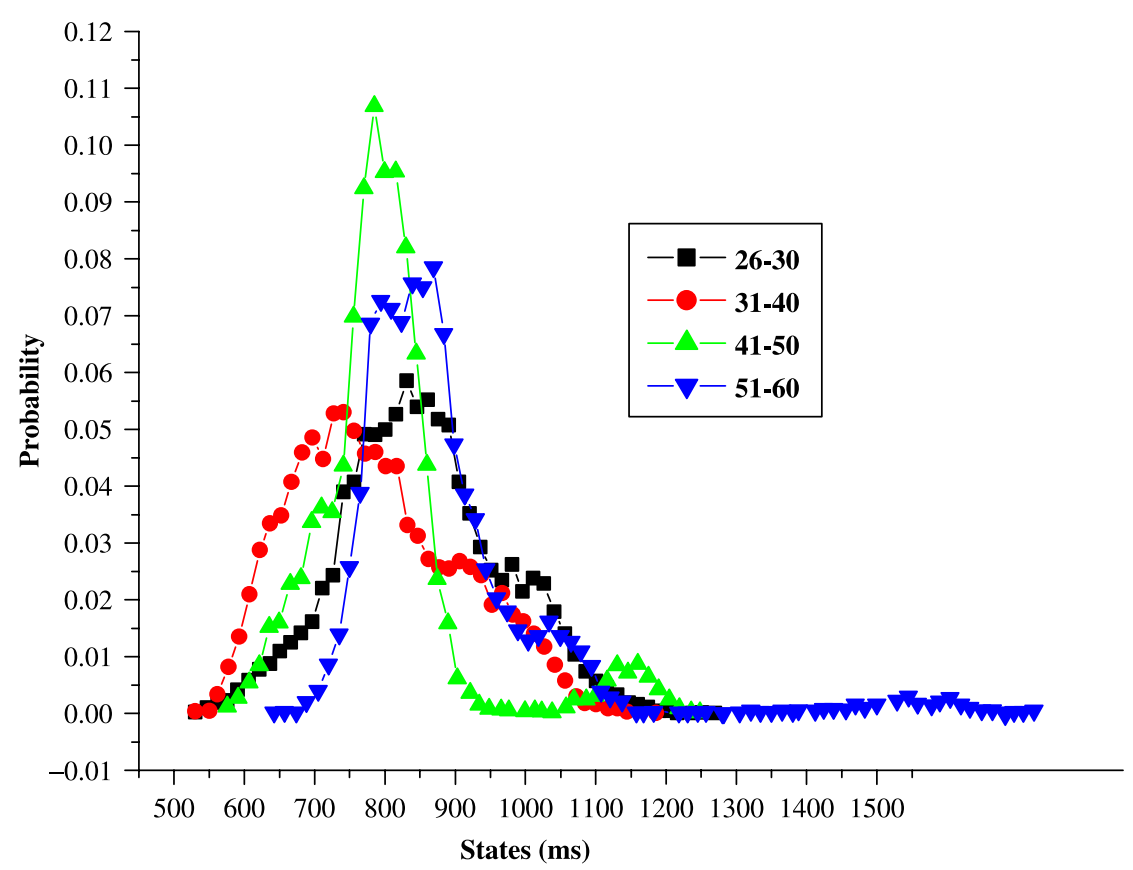

Figure 4. Mean probability distribution of states for each age group.

diminution in other linear index [12,23]. As a consequence of this transition reduction in the 41-50 age group, some transitions disappear with a little increase in the transition with relatively high probability between unstable states as we referred before; these effects reduce the asymmetry values (table 1 ).

In the youngest group, we cannot find a concentration in the transition that could be a good way to distribute the response to excitation between a wide stable states area. However, in the oldest group, the previous reduction in the transition space produces some emerging states with low probability and low transition probability but in a very unstable area.

This effect is a possible explanation for the small increase in the states number, SDRR and entropies. It is not an indicator of complexity increase, but the asymmetry reduction is accordance with a physiological complexity decrease.

It is possible that the mechanism that follows the system during aging changes in oldest subjects with the appearance of a random component in the time series, as this random component increases the signal complexity. This would increases the state number and the entropy [33] but decrease the physiological complexity and reduce at the same time the asymmetry of $\langle F\rangle$.

From a physiological perspective, we saw previously that in the aging the sympathetic tone increases. The reasons of this change remain unclear and could be by multifactorial causes. However, is generally accepted that with the increment of age or in disease like arrhythmias, the conduction mechanism as well as automatism and impulse genesis are very affected by the change in Gap conduction proteins distribution and the heterogeneity of SA node structure [39-41].

The reduction conduction protein concentration like $\mathrm{Cx} 40$ and $\mathrm{Cx} 43$ together with an increment in the sympathetic tone (and surely several other factors) would be associated with an increment in the conduction path and a loss of uniformity in the SA node pulse given 

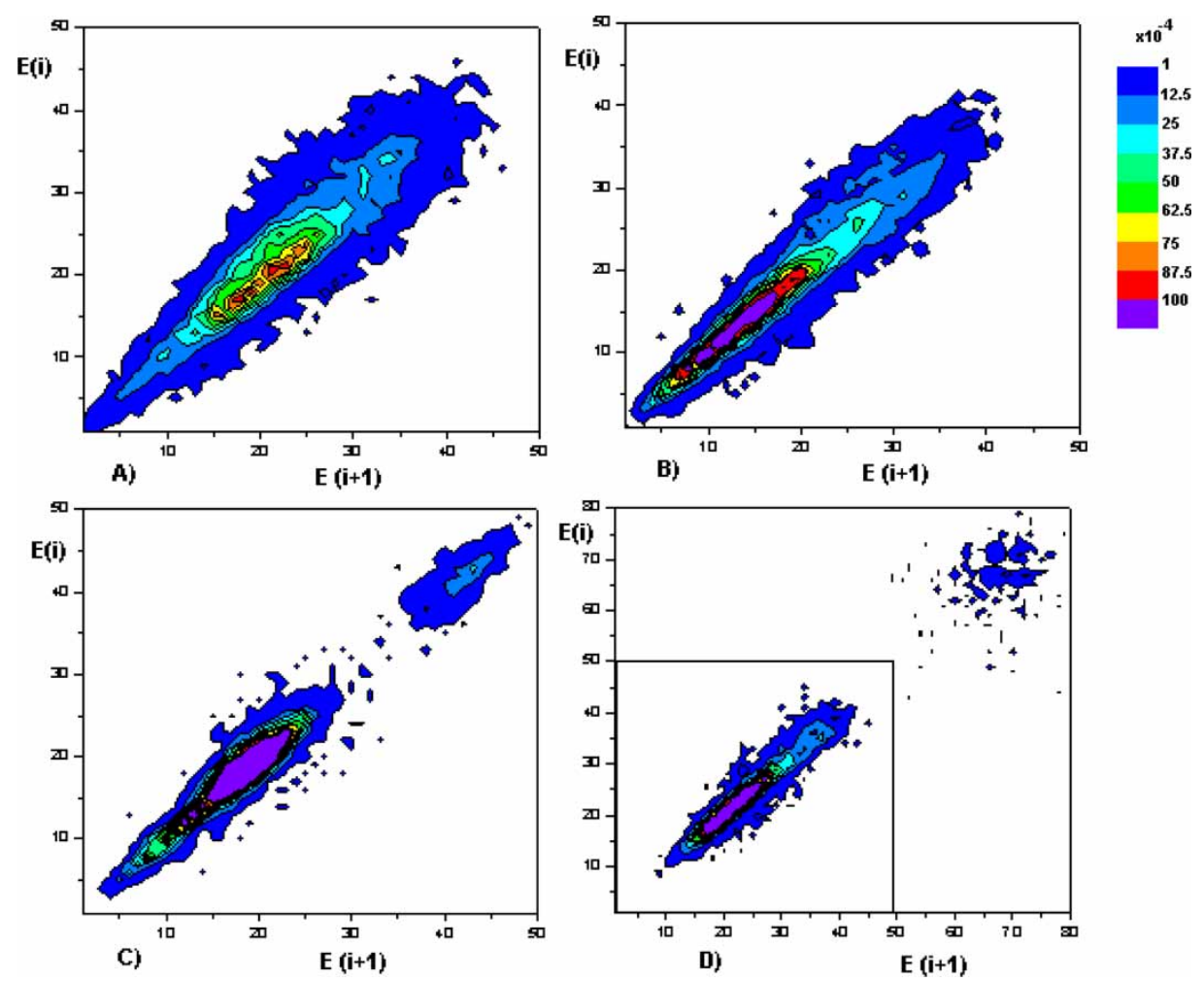

Figure 5. Graphic representation of matrix $\langle F\rangle$ (normalized) according to the ages groups. (A) 26-30, (B) 31-40, (C) 41-50 and (D) 51-60. We applied the same color scale in all cases.

a possible explanation for the reduction in the transition space (roughness) with a small tendency to follow the same transition path in different times scales (loss of complexity) and the increment in the probability of transition to low beat frequency states.

\section{Conclusions}

In this work, we apply a method to the study of the heart rate variability in healthy subjects with different ages using short length records and give an alternative way to the physiological complexity loss.

Our results show that in aging, the capability response decreases and this behavior is associated with the physiological complexity reduction of the system dynamic that could be evaluated with the space transition changes. The emergence of new states and transitions in the oldest group, which correspond to a lower beat frequency, is an interesting result that should be studied later from physiological and mathematical perspectives.

\section{References}

[1] Medvedev, Z.A., 1990, An attempt at a rational classification of theories of ageing, Biological Reviews of the Cambridge Philosophical Society, 65(3), 375-98.

[2] Harman, D., 1986, Free Radicals, Aging and Degenerative Diseases (Alan R. Liss. Inc.), 3-49. 
[3] Task Force of The European Society of Cardiology and The North American Society of Pacing and Electrophysiology (1996) Standards of measurement, physiological interpretation, and clinical use, Circulation, 93(5), 1043-1065.

[4] Huikuri, H.V., Makikallio, T.H. and Perkiomaki, Juha, 2003, Measurement of heart rate variability by methods based on nonlinear dynamics, Journal of Electrocardiology, 36(Supplement).

[5] Kantz, H. and Schreiber, T., 2000, Nonlinear Time Series Analysis (Cambridge: Cambridge University Press).

[6] Corinoa, V.D.A., Matteuccib, M., Cravelloc, L., Ferraric, E., Ferrarid, A.A. and Mainardia, L.T., 2006, Longterm heart rate variability as a predictor of patient age, Computer Methods and Programs in Biomedicine, 82(3), 248-257.

[7] Pincus, S.M., 1991, Approximate entropy as a measure of system complexity, PNAS, 88, 2297-2301.

[8] Costa, M., Peng, C.K., Goldberger, A.L. and Hausdor, J.M., 2003, Multiscale entropy analysis of human gait dynamics, Physica A, 330, 53-60.

[9] Shono, H., Peng, C.K., Goldberger, A.L., Shono, M. and Sugimori, H., 2000, A new method to determine a fractal dimension of non-stationary biological time-serial data, Computers in Biology and Medicine, 30, 237-245.

[10] Salazar, C., Torres, J. and Nieto-Villar, J.M., 2004, Non-linear analysis approach of maternal heart rate patterns in normal and pre-eclamptic pregnancies, Journal of Theoretical Medicine, 5, 219-226.

[11] Makikallio, T.H., Seppanen, T., Juhani Airaksinen, K.E., Koistinen, J., Tulppo, M.P., Peng, C-K., Goldberger, A.L. and Huikuri, H.V., 1998, Heart rate dynamics in patients with stable angina pectoris and utility of fractal and complexity measures, The American Journal of Cardiology, 81, 27-31.

[12] Wood, R., Brian Maraj1, C., Lee, M. and Reyes, R., 2002, Short-term heart rate variability during a cognitive challenge in young and older adults, Age and Ageing, 31, 131-135.

[13] Machado, A., Migliaro, E., Contreras, P. and Coro, F., 2000, Automatic filtering of RR intervals for heart rate variability analysis, Annals of Noninvasive Electrocardiology, 5(3), 255-261.

[14] Schulte-Frohlinde, V., Ashkenazy, Y., Ivanov, P.C., Glass, L., Goldberger, A.L. and Stanley, H.E., 2001, Noise effects on the complex patterns of abnormal heartbeats, Physical Review Letters, 87, 6.

[15] Voss, A., Wessel, N., Kleiner, H.J., Jurths, J. and Dietz, R., 1997, Nonlinear dynamics in cardiovascular diseases, Nonlinear Analysis, Theory, Methods and Applications, 30(2), 935-941.

[16] Voss, A., Jurths, J., Kleiner, H.J., Witt, A., Wessel, N., Saparin, P., Osterziel, K.J., Schurath, R. and Dietz, R., 1996, The application of methods of non-lineal dynamics for the improved and predictive recognition of patients threatened by sudden cardiac death, Cardiovascular Research, 31, 419-433.

[17] Yang, A.C.C., Hseu, S-S., Yien, H-W., Goldberger, A.L. and Peng, C.K., 2003, Linguistic analysis of the human heartbeat using frequency and rank order statistics, Physical Review Letters, 90(10).

[18] Daw, C.S., Finney, C.E.A. and Tracy, E.R., 2002, A review of symbolic analysis of experimental data. Available from: http://www-chaos.engr.utk.edu/pap/cgr-rsi2002.pdf

[19] Barbieri, R., Matten, E.C., Alabi, A.A. and Brown, E.N., 2005, A point-process model of human heartbeat intervals: new definitions of heart rate and heart rate variability, American Journal of Physiology, Heart and Circulatory Physiology, 288, 424-435.

[20] Stanley, G.B., Poolla, K. and Siegel, R.A., 2000, Threshold modeling of autonomic control of heart rate variability, IEEE Transactions on Biomedical Engineering, 47, 1147-1153.

[21] Goldberger, A.L., 2001, Heartbeats, hormones and health. Is variability the spice of life, The American Journal of Respiratory and Care Medicine, 163, 1289.

[22] Ivanov, P.Ch., Rosenblum, M.G., Peng, C.K., Mietus, J.E., Havlin, S., Stanley, H.E. and Goldberger, A.L., 1998, Scaling and universality in heart rate variability distributions, Physica A, 249, 587-593.

[23] Rajendra, A.U., Kannathal, N., Sing, O.W., Ping, L.Yi. and Chua, T., 2004, Heart rate analysis in normal subjects of various age groups, Biomedical Engineering Online, 3, 24.

[24] Pinto, D.S., Ho, K.K., Zimetbaum, P.J., Pedan, A. and Goldberger, A.L., 2003, Sinus versus nonsinus tachycardia in the emergency department: importance of age and heart rate, BMC Cardiovascular Disorders, 3.

[25] Nikhil, I., Peng, C.K., Goldberger, A.L. and Lipsitz, L., 1996, Age-related alterations in the fractal scaling of cardiac interbeat interval dynamics, The American Journal of Physiology, 27(1), 1078-1084.

[26] Stein, P.K., Kleiger, R.E. and Rottman, J.N., 1997, Differing effects of age on heart rate variability in men and women, The American Journal of Cardiology, 80, 302-305.

[27] Terry Kuo, B.J., Tsann, L., Cheryl Yang, C.H., Chia-Lin, L., Chieh-Fu, C. and Pesus, C., 1999, Effect of aging on gender differences in neural control of heart rate, The American Journal of Physiology, 46, 2233-2239.

[28] Antelmi, I., Paula, R.S.D., Shinzato, A.R., Peres, C.A., Mansur, A.J. and Grupi, C.J., 2004, Influence of age, gender, body mass index, and functional capacity on heart rate variability in a Cohort of subjects without heart disease, The American Journal of Cardiology, 93, 381-385.

[29] Brennan, M., Palaniswami, M. and Kamen, P., 2001, Do existing measures of Poincaré plot geometry reflect nonlinear features of heart rate variability?, IEEE Transactions on Biomedical Engineering, 48(11).

[30] Brennan, M., Palaniswami, M. and Kamen, P., 2002, Poincaré plot interpretation using a physiological model of HRV based on a network of oscillators, American Journal of Physiology, Heart and Circulatory Physiology, 283, $1873-1886$. 
[31] Kamen, P.W., Krum, H. and Tonkin, A.M., 1996, Poincare plot of heart rate variability allows quantitative display of parasympathetic nervous activity, Clinical Science, 91, 201-208.

[32] Ivanov, P.C., Amaral, L.A., Goldberger, A.L, Havlin, S., Rosenblum, M.G., Struzikk, Z.R. and Stanley, H.E., 1999, Multifractality in human heartbeat dynamics, Nature, 399, 3.

[33] Goldberger, A.L., Peng, C.K. and Lipsitz, L.A., 2002, Why is physiologic complexity and how does it change with aging and disease?, Neurobiology of Aging, 23, 23-26.

[34] Lin, J., Keogh, E., Lonardi, S. and Chiu, B., 2003, A Symbolic Representation of Time Series, with Implications for Streaming Algorithms, In proceedings of the 8th ACM SIGMOD Workshop on Research Issues in Data Mining and Knowledge Discovery.

[35] Beckers, F., Verheyden, B. and Aubert, A.E., 2006, Aging and nonlinear heart rate control in a healthy population, American Journal of Physiology, Heart and Circulatory Physiology, 290, 2560-2570.

[36] Huikuri, H.V., Kessler, K.M., Terracall, E., Castellanos, A., Linnaluoto, M.K. and Myerburg, R.J., 1990, Reproducibility and circadian rhythm of heart rate variability in healthy subjects, The American Journal of Cardiology, 65, 391-393.

[37] Laitinen, T., Hartikainen, J., Vanninen, E., Niskanen, L., Geelen, G. and Länsimies, E., 1998, Age and gender dependency of baroreflex sensitivity in healthy subjects, The American Journal of Physiology.

[38] Tasaki, H., Serita, T., Ueyama, C., Kitano, K., Seto, S. and Yano, K., 2006, Long-term follow-up of the circadian rhythm of heart rate and heart rate variability in healthy elderly patients, Circulation Journal, 70, 889-895.

[39] Jones, S.A., Lancaster, M.K. and Boyett, M.R., 2004, Ageing-related changes of connexins and conduction within the sinoatrial node, The Journal of Physiology, 560(2), 429-437.

[40] Kanagaratnam, P., Rothery, S., Patel, P., Severs, N.J. and Peters, N.S., 2002, Relative expresion of immunolocalized connexins 40 and 43 correltes with human atrial conduction properties, Journal of the American College of Cardiology, 39, 116-123.

[41] Rubart, M. and Zipes, D.P., 2005, Mechanisms of sudden cardiac death, The Journal of Clinical Investigation, $\mathbf{1 1 5}, 2305-2315$ 


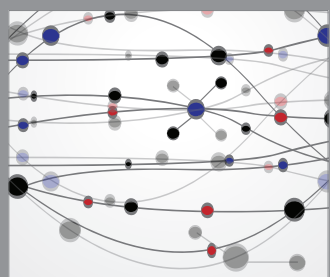

The Scientific World Journal
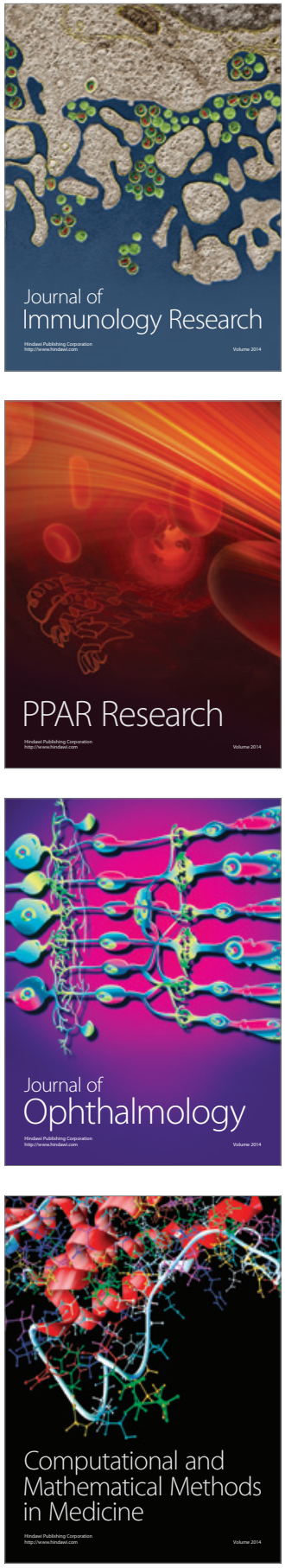

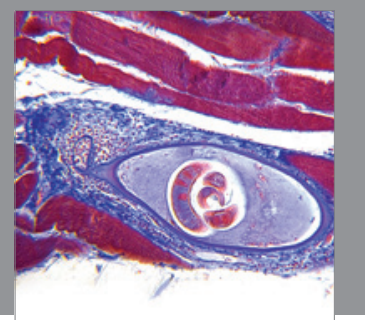

Gastroenterology

Research and Practice
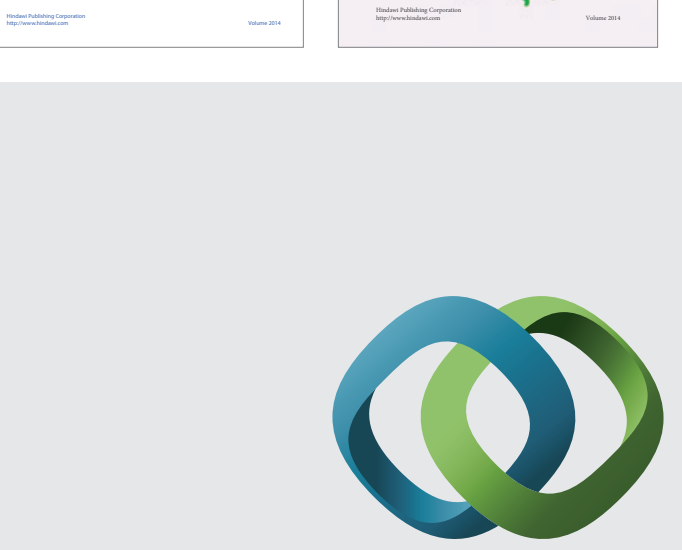

\section{Hindawi}

Submit your manuscripts at

http://www.hindawi.com
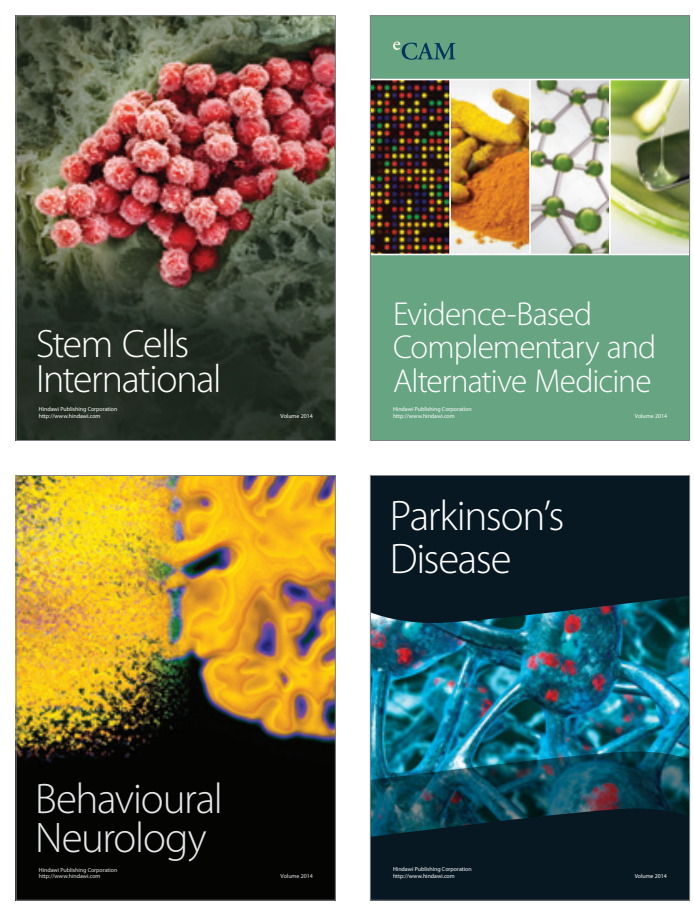

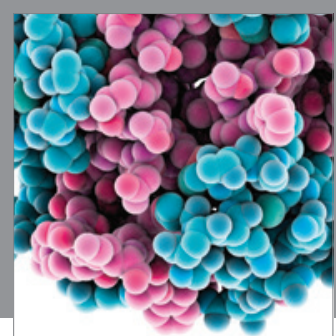

Journal of
Diabetes Research

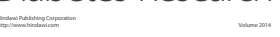

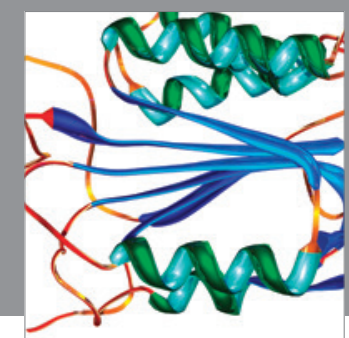

Disease Markers
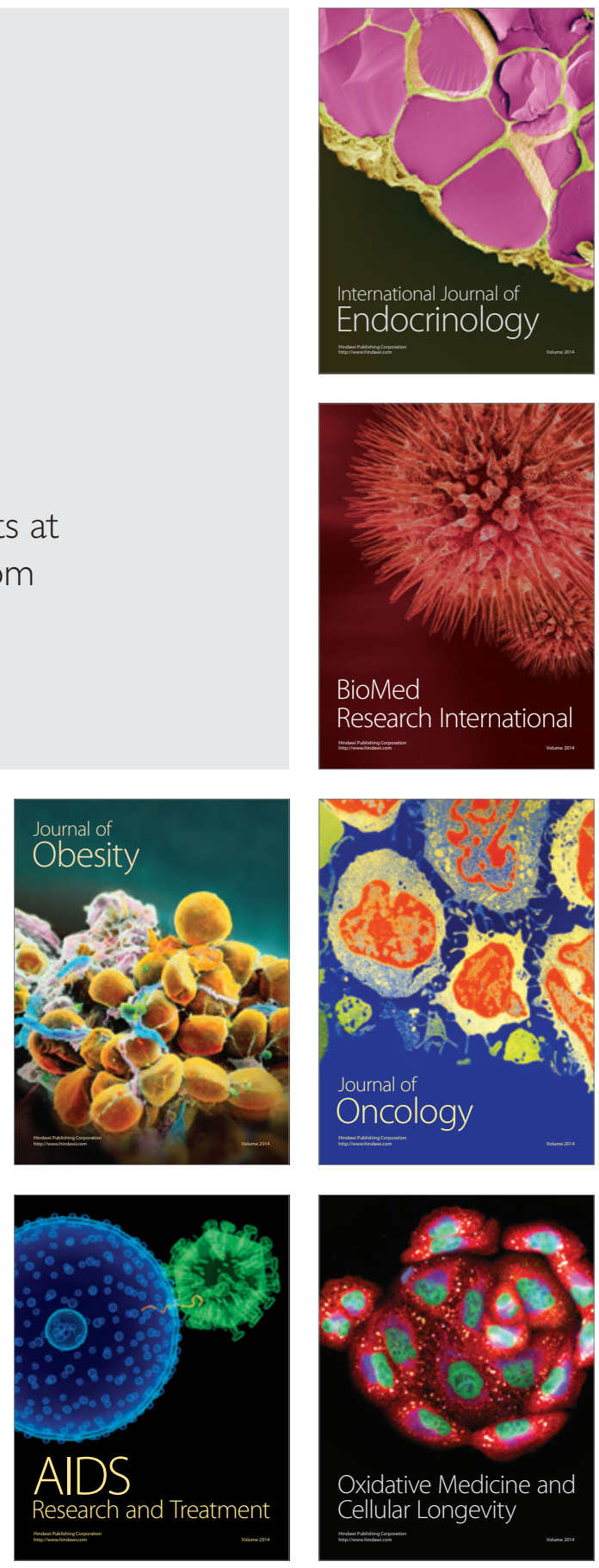